\title{
Aspects réglementaires et organisationnels de l'activité d'autoconservation. Le point de vue du juriste
}

\author{
Anna GRABINSKI \\ Faculté de droit, Université Paris 5 René Descartes, Malakoff
}

\begin{abstract}
RESUME
L'activité d'autoconservation n'est, à l'heure actuelle, abordée que de manière incidente dans des textes d'application des lois de bioéthique. Les conditions de sa mise en œuvre diffèrent selon la nature de l'élément conservé : alors que l'autoconservation d'ovocytes et de tissu ovarien relèverait de la recherche biomédicale, l'autoconservation de sperme serait assimilée à une activité d'assistance médicale à la procréation.

L'activité d'autoconservation se trouve consacrée dans le projet de révision des lois de bioéthique.

Les éléments ainsi conservés peuvent être restitués au seul déposant. Ils peuvent également être utilisés, soit pour une assistance médicale à la procréation, qui est la finalité première de l'autoconservation, soit dans le cadre d'une recherche. Suite à une utilisation partielle, ou en l'absence d'une telle utilisation, la destruction des éléments autoconservés peut être envisagée, ainsi que la poursuite de l'autoconservation. Ces hypothèses posent toutefois des difficultés n'ayant pas été résolues dans le projet de loi relatif à la bioéthique.
\end{abstract}

Mots-clés : paillettes de sperme, ovocytes, tissu ovarien, recherche biomédicale, assistance médicale à la procréation, restitution, destruction, poursuite de la conservation

\section{INTRODUCTION}

L'étude des règles juridiques ayant trait à l'activité d'autoconservation s'entend bien entendu de l'analyse de la réglementation actuelle; mais l'on ne saurait envisager une telle étude sans aborder également les évolutions apportées par le projet de loi relatif à la bioéthique, adopté en seconde lecture par l'Assemblée Nationale le 11 décembre 20031 .
Dans un premier temps, nous aborderons la question des conditions de mise en œuvre de l'activité d'autoconservation - il s'agira, en effet, de savoir dans quel cadre juridique cette activité s'inscrit-elle à l'heure actuelle, quelles règles, issues de quels textes, doivent-elles être respectées pour pouvoir y procéder.

Dans un second temps, nous nous interrogerons sur le devenir des éléments autoconservés - cette étude portera sur les différentes hypothèses envisageables (leur restitution, les modes d'utilisation, l'éventuelle destruction ou la poursuite de la conservation), ainsi que sur les conditions dans lesquelles elles peuvent être effectuées.

\section{LES CONDITIONS DE MISE EN CEUVRE DE L'AUTOCONSERVATION}

\section{A.L'ACTIVITE D'AUTOCONSERVATION DANS LA REGLEMENTATION ACTUELLEMENT EN VIGUEUR}

\section{Les dispositions relatives à l'activité d'autoconservation}

L'autoconservation ne fait pas l'objet, dans les lois de bioéthique, de règles spécifiques.

Elle n'est ni expressément interdite, ni formellement autorisée.

Malgré le silence du législateur, elle a cependant été abordée, de manière incidente, au sein de deux textes d'application des lois de bioéthique.

II s'agit, d'une part, de l'arrêté du 10 février 1998 fixant le contenu du rapport annuel d'activités des établissements ou laboratoires autorisés à pratiquer des activités d'assis-

\section{Correspondance :}

Anna GRABINSKI - ATER, Faculté de droit, Université Paris 5 René Descartes, Centre de Recherches en droit médical, 10, avenue Pierre Larousse 92245 MALAKOFF cedex - Tel 06.20.66.47.86 - Email a.grabinski@free.fr 
tance médicale à la procréation (point F), quil impose la déclaration du bilan des autoconservations de sperme se rapportant non seulement à la mise en œuvre d'une assistance médicale à la procréation, mais également à des traitements stérilisants ou des vasectomies.

D'autre part, et surtout, l'arrêté du 12 janvier 1999 relatif aux bonnes pratiques cliniques en assistance médicale à la procréation, décrit, dans un point $2.6^{2}$, les conditions d'ordre médical, biologique et juridique, de la mise en cuvre d'une autoconservation de gamètes ou de tissu gonadique. Une distinction est établie selon la nature de l'élément prélevé : alors que l'autoconservation de sperme est considérée comme une pratique courante, celle des ovocytes et du tissu ovarien doit s'inscrire dans un protocole de recherche biomédicale, soumis dès lors aux dispositions de la loi Huriet du 20 décembre 1988 (articles L.1121-1 à L.1126-7 du Code de la santé publique).

\section{Les difficultés liées à l'assimilation de l'autoconser- vation des ovocytes et de tissu ovarien à une recher- che biomédicale}

II résulte du point 2.6 de l'arrêté du 12 janvier 1999 que le prélèvement et la conservation, pour une éventuelle assistance médicale à la procréation ultérieure, d'ovocytes et de tissu ovarien doivent faire l'objet d'une recherche biomédicale, et sont soumis à ce titre aux dispositions de la loi Huriet du 20 décembre 1988 (articles L. 1121-1 et suivants du Code de la santé publique).

Les difficultés liées à cette qualification tiennent non seulement à l'impossibilité de déterminer les actes (prélèvement, cryoconservation, utilisation) devant être concernés par un protocole de recherche, mais encore à la nature mixte de la démarche d'autoconservation de tissu ovarien et d'ovocytes, qui se situe à mi-chemin entre la recherche et le soin. En effet, l'autoconservation de tissu ovarien est, non seulement, et dans une certaine mesure, expérimentale, en raison des incertitudes quant à la préservation effective de la fertilité, mais encore partiellement préventive, puisque face à un risque de perte de fertilité, il existe une volonté et un espoir de préserver cette dernière pour la personne ellemême. Or, ces deux domaines de l'activité médicale diffèrent par leur finalité : alors que la recherche biomédicale a pour fin le développement des connaissances médicales ou biologiques, le soin a pour objet des actes effectués pour la personne elle-même.

Loin de clarifier une situation déjà broussailleuse et empreinte d'incertitude, le point 2.6 de l'arrêté du 12 janvier 1999 ajoute encore à cette complexité : sa rédaction laisse en effet place à deux hypothèses parfaitement distinctes, qui ne sont pourtant pas exclusives l'une de l'autre.

Soit, d'une part, l'on considère que le protocole de recherche porte non seulement sur la conservation, mais également sur l' " utilisation procréative " des éléments prélevés.

On ne peut, dès lors, employer le terme d' " autoconservation ", puisque l'objet du protocole de recherche n'est pas, dans cette hypothèse, la garde, pour une personne, de son potentiel procréatif, mais la seule utilisation expérimentale de fragments préalablement cryoconservés.
Soit, d'autre part, l'on admet que seule l'autoconservation doit faire l'objet d'un protocole de recherche, l'éventuelle assistance médicale à la procréation n'étant que la finalité et la condition de la mise en œuvre de cette conservation. Dans cette hypothèse, c'est la qualification de recherche biomédicale qui est inadaptée : en effet, la seule finalité d'une recherche biomédicale est le développement des connaissances médicales ou biologiques.

Or, une autoconservation, qui est la garde, pour une personne, de son potentiel procréatif, est un acte effectué exclusivement pour la personne elle-même, ce qui le situe donc dans une démarche de soin et non de recherche.

Comment, dès lors, concilier ces deux aspects d'une même démarche, et se conformer ainsi aux exigences réglementaires du point 2.6 de l'arrêté du 12 janvier 1999 ?

3. La contestable compatibilité des dispositions réglementaires relatives à l'activité d'autoconservation de sperme avec les dispositions légales relatives à l'assistance médicale à la procréation

\section{a) L'assimilation de l'activité d'autoconservation de sperme à une activité d'assistance médicale à la pro- création}

Les textes réglementaires d'application des dispositions légales relatives à l'assistance médicale à la procréation (issues des lois de bioéthique) traitent des actes effectués en vue ou au cours d'une tentative d'assistance médicale à la procréation.

Formant un ensemble dans la mise en œuvre d'une assistance médicale à la procréation, tous ces actes seraient donc soumis aux conditions légales d'accès à cette activité régie par les articles L. $2141-1$ et suivants du Code de la santé publique.

En faveur d'une telle interprétation, on peut en effet se référer à la définition large de l'assistance médicale à la procréation, donnée à l'article L.2141-1 du Code de la santé publique : elle s'entend « des pratiques cliniques et biologiques permettant la conception in vitro, le transfert d'embryons et linsémination artificielle, ainsi que de toute technique d'effet équivalent permettant la procréation en dehors du processus naturel ». Or, l'autoconservation permet également une future procréation dans le cadre d'une AMP.

De plus, les règles relatives à l'autoconservation de gamètes et de tissu gonadique figurant précisément au sein de ces textes réglementaires d'application (arrêté de 1998 et arrêté de 1999 précités), on peut donc en déduire l'assimilation de l'activité d'autoconservation à une activité d'assistance médicale à la procréation.

Il s'agirait, enfin, d'une activité d'assistance médicale à la procréation intraconjugale - l'autoconservation est, en effet, abordée au sein d'un point 2 de l'arrêté du 12 janvier 1999 intitulé "Description des pratiques d'AMP intraconjugale".

b) Une telle assimilation équivaudrait pourtant à l'impossibilité de sa mise en cuvre

Cette impossibilité tient principalement aux conditions et 
aux indications de l'assistance médicale à la procréation, telles qu'elles résultent de l'article L.2141-2 du Code de la santé publique.

En effet, et surtout, l'infertilité à laquelle l'assistance médicale à la procréation est destinée à remédier doit être médicalement diagnostiquée, ce qui n'est pas le cas en l'espè$c e$, puisque l'individu auquel une autoconservation est proposée est encore fertile.

De plus, l'assistance médicale à la procréation ne peut s'adresser qu'à un couple (marié ou vivant en concubinage depuis au moins deux ans), en âge de procréer, ayant un projet parental concret et actuel. Or, l'autoconservation, dont la finalité est de tenter de préserver la fertilité compromise d'une personne, s'adresse indifféremment aux majeurs et mineurs, aux personnes célibataires et vivant en couple, avec ou sans projet parental. Dès lors, elle peut concerner (et concerne le plus souvent) des personnes ne répondant pas à l'ensemble de ces conditions d'accès à l'assistance médicale à la procréation.

\section{c) Une autre interprétation semble toutefois possible}

Bien qu'introduites au sein de la partie consacrée à l'assistance médicale à la procréation intraconjugale dans l'arrêté du 12 janvier 1999, les dispositions relatives à l'autoconservation opèrent une distinction entre, d'une part, une autoconservation en vue d'une éventuelle assistance médicale à la procréation ultérieure et, d'autre part, une autoconservation dans le cadre d'une tentative d'assistance médicale à la procréation déjà mise en œuvre, parce que répondant à la demande parentale d'un couple.

- Dans la première hypothèse, l'autoconservation se situe en-dehors d'une assistance médicale à la procréation

L'autoconservation est effectuée en vue d'une assistance médicale à la procréation ultérieure : elle se situe donc endehors d'une quelconque tentative actuelle d'assistance médicale à la procréation, cette dernière n'étant que l'une des utilisations possibles, et futures, du sperme autoconservé.

Le respect des conditions légales relatives à l'assistance médicale à la procréation ne pourra alors être exigé qu'au moment et dans le cas où le déposant de l'élément autoconservé souhaitera entrer dans ce «processus procréatif $"$.

Point 2.6.1 §1 : " L'autoconservation de sperme doit être systématiquement proposée aux patients avant toute thérapeutique ou intervention potentiellement sterilisante (chimiothérapie, radiothérapie, chirurgie...) ".

- Dans la seconde hypothèse, en revanche, l'autoconservation est envisagée alors qu'une procédure d'assistance médicale à la procréation a déjà été entamée.

Le respect des dispositions légales relatives à l'assistance médicale à la procréation ne conditionnerait ici que la mise en œuvre de la tentative d'assistance médicale à la pro- création, et non pas la possibilité de l'autoconservation. Cette dernière ne serait qu'un acte parmi d'autres, facultatif au demeurant, inclus au sein d'un " processus procréatif préexistant " .

Point 2.6.1 §2 : " Elle peut aussi être préconisée avant ou au cours d'une tentative d'AMP 》.

Une telle interprétation semble conforme au point 2.6.1 alinéa 7 de l'arrêté, qui dispose :

"L'utilisation ultérieure du sperme en vue d'un acte d'assistance médicale à la procréation ne pourra se faire que dans la mesure où seront remplies les conditions prévues par l'article L. 152-2 du code de la santé publique ".

Face à ces incertitudes, contradictions et difficultés, le législateur est intervenu et a consacré l'activité d'autoconservation, dans le projet de loi relatif à la bioéthique, par l'élargissement des indications de l'assistance médicale à la procréation.

B. LA CONSECRATION, DANS LE PROJET DE LOI RELATIF A LA BIOETHIQUE, DE L'ACTIVITE D'AUTOCONSERVATION PAR L'ELARGISSEMENT DES INDICATIONS DE L'ASSISTANCE MEDICALE A LA PROCREATION

1. L'article L. 2141-11 du projet de loi relatif à la bioéthique, adopté en seconde lecture par l'Assemblée Nationale le 11 décembre 2003

Consacrée à l'article L. 2141-11 du projet ${ }^{3}$, qui la prévoit expressément, l'autoconservation de gamètes et de tissu gonadique sera rendue possible pour toute personne répondant aux indications prévues par la loi (prise en charge médicale susceptible d'altérer sa fertilité ou risque d'altération prématurée de la fertilité), indépendamment de son âge et de sa situation familiale, sous réserve de son consentement ou de celui de l'un des titulaires de l'autorité parentale ou encore du tuteur s'il s'agit d'un mineur ou majeur sous tutelle.

La consécration législative n'a cependant pas résolu toutes les difficultés.

2. Les difficultés résultant d'un champ d'application large de l'article L.2141-11 du projet de révision

On peut se demander si l'autoconservation systématique de tissu gonadique (et nous songerons notamment au tissu ovarien) est réellement justifiée alors même que son utilisation, malgré des avancées incontestables, n'est encore qu'en cours de validation.

En effet, ce qui permet de "légaliser " l'autoconservation de sperme est le fait qu'en cas de souhait de la personne de recourir à une assistance médicale à la procréation, cette dernière est possible. II y a donc une utilité de l'autoconservation en vue d'une éventuelle assistance médicale à la procréation ultérieure, puisque le but de l'autoconservation pourra être réalisé.

Or, ceci n'est pas vrai pour l'autoconservation de tissu ovarien : il y a un risque d'inutilité de cette autoconservation, en 
ce que l'utilisation de ce prélèvement n'est pas encore parfaitement au point, malgré les progrès récents en matière d'utilisation à visée procréative du tissu ovarien autoconservé - aussi, y a-t-il un réel intérêt de l'autoconservation, proposée systématiquement, justifiant l'atteinte à l'intégrité corporelle de la personne sur laquelle le prélèvement est effectué ? 4

Ceci amène donc à s'interroger sur le devenir des éléments autoconservés : outre leur possible restitution au déposant, leur utilisation, soit pour une assistance médicale à la procréation (finalité première de l'autoconservation), soit dans le cadre d'une recherche, peut être envisagée. De même, et à certaines conditions, une éventuelle destruction de ces éléments peut être décidée, tout comme une éventuelle poursuite de leur conservation.

\section{LE DEVENIR DES ELEMENTS AUTOCONSERVES}

\section{A. LE PRINCIPE DE RESTITUTION AU SEUL DEPO- SANT DES PAILLETTES AUTOCONSERVEES}

Dans la réglementation actuellement en vigueur, cette question n'est soulevée qu'à propos de l'autoconservation de sperme dans la mesure où, rappelons-le, l'autoconservation de tissu ovarien et d'ovocytes doit faire l'objet d'un protocole de recherche biomédicale.

Ainsi, les paillettes sont-elles restituées, sur sa demande expresse, au seul déposant, lequel devra alors signer un document, contresigné par le médecin biologiste.

Cela découle de l'arrêté du 12 janvier 1999 qui dispose, dans un point 2.6.1, §6 :

"Seul le déposant peut demander la restitution (...) de ses paillettes. Dans ces cas, un document est alors signé parle patient et contresigné par le médecin biologiste ".

Dès lors, nulle personne, en-dehors du déposant lui-même, ne peut demander la restitution des éléments autoconservés - l'épouse, la compagne, les membres de la famille sont également considérés comme des tiers et ne peuvent donc prétendre à une telle restitution.

Suite au décès du déposant, a fortiori, la disposition précitée fait également obstacle à toute remise à un tiers des paillettes autoconservées.

II est enfin fort probable que le principe de la non - restitution des paillettes à un tiers, résultant de l'arrêté du 12 janvier 1999, soit reconduit dans les textes d'application de la loi de bioéthique révisée, et étendu aux tissus gonadiques et ovocytes.

\section{B. L'UTILISATION DES ELEMENTS AUTOCONSERVEES}

1. L'assistance médicale à la procréation : finalité première de l'autoconservation

a) Du vivant de la personne.

La finalité première de l'autoconservation est l'assistance médicale à la procréation, qui ne pourra cependant être mise en cuvre que dans le respect des conditions légales de l'article L.2141-2 du Code de la santé publique ${ }^{5}$.

On songe ici principalement à l'utilisation de sperme, puisque les techniques d'assistance médicale à la procréation à partir de tissu ovarien ou d'ovocytes, bien qu'ayant donné lieu, très récemment, à des résultats fort encourageants, n'en sont pas moins encore en cours de validation.

Or, ce n'est qu'au terme de ce processus de validation des techniques, effectué par le biais d'une recherche sur " l'utilisation procréative " des fragments autoconservés - soit au sein du même protocole que l'autoconservation, soit dans le cadre d'un protocole distinct -, que l'on pourrait avoir recours de manière systématique à une autoconservation.

II convient en outre de souligner que le projet de loi relatif à la bioéthique, tout en consacrant l'activité d'autoconservation à l'article L.2141-11, quelle que soit la nature de l'élément autoconservé, consacre par la même l'assistance médicale à la procréation comme finalité première de l'autoconservation.

En effet, cet article dispose : « En vue de la réalisation ultérieure d'une assistance médicale à la procréation 6 , toute personne peut bénéficier du recueil et de la conservation de ses gamètes ou de tissu germinal, avec son consentement et, le cas échéant, celui de l'un des titulaires de l'autorité parentale, ou du tuteur lorsque l'intéressé mineur ou majeur fait l'objet d'une mesure de tutelle, lorsqu'une prise en charge médicale est susceptible d'altérer sa fertilité, ou lorsque sa fertilité risque d'être prématurément altérée ".

\section{b) Après le décès de la personne}

L'assistance médicale à la procréation n'est plus envisageable après le décès de la personne, ni au vu de la réglementation actuellement en vigueur, ni dans le projet de loi, les deux membres du couple devant en effet être vivants (article L.2141-2 du Code de la santé publique).

\section{La recherche sur les éléments autoconservés}

Une recherche - qui ne serait pas nécessairement biomédicale - semble possible sur un élément autoconservé et ce, quels que soient sa nature et le moment de sa mise en œuvre (du vivant de la personne ou après son décès).

Sa mise en œuvre devrait toutefois être soumise à l'accord préalable et exprimé du vivant de la personne dont les éléments ont été conservés : on peut en effet penser que le caractère personnel de la conservation serait un obstacle absolu à toute utilisation en dehors d'un tel accord.

Une telle utilisation est expressément rendue possible dans le projet de révision : selon l'article L.1211-27 du projet, après avoir fait l'objet de mesures de conservation - telles la congélation - la réutilisation à une fin scientifique ou médicale différente de la fin initiale ${ }^{8}$ pourra être envisagée dans toutes les hypothèses où un prélèvement aura été initialement effectué ou une collection constituée, dans un 
but médical ou de recherche (qu'elle soit, ou non, biomédicale).

La condition d'un tel changement de finalité est l'information préalable et l'absence d'opposition de la personne.

Contrairement aux autres tissus, cellules et produits, le projet de loi n'a prévu, pour les gamètes et les tissus gonadiques, aucune exception à l'exigence de manifestation de volonté de l'intéressé.

De même, un changement de finalité n'est plus possible après le décès de la personne.

\section{DESTRUCTION OU POURSUITE DE LA CONSERVATION ?}

\section{Les difficultés dans les dispositions actuellement en vigueur}

A l'heure actuelle, ces hypothèses ne sont prévues que pour le sperme, il est donc difficile de se prononcer sur l'éventualité d'une destruction ou de la poursuite de la conservation de tissu ovarien ou d'ovocytes autoconservés.

Toute la difficulté réside dans la contradiction existant entre deux dispositions de l'arrêté du 12 janvier 1999 et les difficultés liées à leur interprétation.

Selon le point $2.6 .1, \S 5$ : « La durée de la conservation est prévue pour un an renouvelable sur demande écrite".

Si l'on interprète " a contrario " cette disposition, il semblerait donc que la destruction d'office soit possible à l'expiration du délai si le déposant n'a pas demandé expressément la poursuite de la conservation.

Cependant, selon le point 2.6.1, §6 : "Seul le déposant peut demander (...) la destruction de ses paillettes. Dans ces cas, un document est alors signé par le patient et contresigné par le médecin biologiste ».

Cette disposition n'autoriserait donc la destruction des paillettes qu'à la condition d'une demande exprimée par le déposant lui-même, à l'exclusion de toute autre personne.

Dès lors, selon une première interprétation, cet article empêcherait quiconque, $y$ compris la structure en charge de la conservation (un CECOS, notamment), de décider de la destruction des paillettes et ce, même dans le cas de l'expiration du délai d'un an prévu au point 2.6.1, §5.

Une telle décision serait également prohibée en cas de décès du déposant, puisque la destruction automatique dans cette hypothèse n'est pas expressément prévue par les textes.

La décision appartiendrait donc exclusivement au déposant, et ferait obstacle à toute destruction en-dehors de son accord exprès.

Toutefois, selon une seconde interprétation, le point 2.6.1 $\S$ 6 ne ferait obstacle qu'aux seules demandes de destruction émanant de tiers à la structure en charge de la conserva- tion du sperme, formées auprès de cette dernière quel que soit le moment (au cours de la conservation, ou même à l'écoulement du délai d'un an).

Cette disposition permettrait cependant à une telle structure de décider de la destruction des paillettes à l'expiration du délai d'un an et ce, en l'absence d'une demande (écrite) de poursuite de la conservation formée par le déposant.

\section{Des difficultés non résolues dans le projet de révision des lois de bioéthique}

Le législateur n'a pas souhaité impartir de délai de conservation, au-delà duquel la destruction des éléments autoconservés serait possible.

II en résulte les mêmes difficultés que celles découlant de la réglementation actuellement en vigueur.

De même, aucune disposition du projet de loi ne prévoit la destruction automatique de l'élément autoconservé en cas de décès du déposant. Or, l'utilisation à une autre fin des éléments autoconservés ainsi que la procréation post-mortem étant interdites dans cette hypothèse, on aboutirait dès lors à la poursuite indéfinie de leur conservation.

\section{CONCLUSION}

Prévue en des termes généraux à l'article L.2141-11 du projet de loi relatif à la bioéthique, l'autoconservation de gamètes et de tissu gonadique semble désormais acquise en son principe. La future loi permet également d'unifier le régime juridique de l'autoconservation, en ne le distinguant plus selon la nature de l'élément autoconservé, comme c'est encore le cas dans la réglementation actuellement en vigueur.

Cependant, force est de constater que des incertitudes importantes demeurent, et il serait souhaitable qu'elles soient levées à l'occasion de la révision des lois de bioéthique.

En effet, l'on peut, d'une part, s'interroger sur l'opportunité d'une telle généralisation et de la mise en cuvre systématique d'une pratique qui, dans certains cas (et nous songerons notamment aux autoconservations de tissus ovariens), n'est qu'en cours de validation. D'autre part, un certain nombre de questions et d'interrogations, apparues sous l'empire de la réglementation actuelle et non résolues aujourd'hui, n'ont pourtant pas reçu de réponse législative au sein du projet précité les mêmes difficultés sont dès lors à craindre, venant s'ajouter à celles, particulières, qui résulteront des dispositions nouvelles.

Nota : En discussion à la date de l'intervention dont est issu cet article, la loi relative à la bioéthique a, depuis, été définitivement adoptée. Il s'agit de la loi $n^{\circ} 2004-800$ du 6 août 2004, publiée au JO n'182 du 7 août 2004, $p$. 14040. 


\section{REFERENCES}

1. Arrêté ministériel du 10 février 1998 fixant le contenu du rapport annuel d'activités des établissements ou laboratoires autorisés à pratiquer des activités d'assistance médicale à la procréation. Journal Officiel, 19 février 1998, 2601.

2. Arrêtẻ ministériel du 12 janvier 1999 relatif aux règles de bonnes pratiques cliniques et biologiques en assistance médicale à la procréation. Journal Officiel, 28 février 1999, 3061.

3. Projet de loi relatif à la bioéthique, adopté en seconde lecture par l'Assemblée Nationale, le 11 décembre 2003, http:// uww.assemblee-nationale.fr/12/ta/ta0215.asp

4. Dictionnaire Permanent Bioéthique et Biotechnologies, " Assistance médicale à la procréation (AMP), Editions Législatives, 1 er février 2002, feuillets 28, pp.109-129.

5. GRABINSKI A. : Consentement et examen des caractéristiques génétiques dans le projet de révision des lois de bioéthique. Rapport d'activité 2000-2003 de la Commission consultative nationale en matière d'examens des caractéristiques génétiques à des fins médicales. En cours de publication, ce rapport pourra être consulté en ligne fin 2004 : http://www.sante.gouv.fr

6. OKTAY K., BUYUK E., VEECK I. et al. : Embryo development after heterotopic transplantation of cryopreserved ovarian tissue. Lancet, 2004,363 (9412) : 837-840

7. OKTAY K., BUYUK E. : Fertility preservation in women undergoing cancer treatment. Lancet, 2004, 363 (9423) : 1830.

8. SMITZ J. : Oocyte developmental competence after heterotopic transplantation of cryopreserved ovarian tissue. Lancet, 2004, 363 (9412) : 832-833.

9. TAGLIONE C. : Le recours à l'assistance médicale à la procréation à l'épreuve de la révision des lois de bioéthique. Petites affiches, 17 juin 2003, $120: 4-14$

Communication au Colloque de la Fédération des CECOS, Lyon, 18 mars 2004.

Préserver la fertilité des patients soumis à des traitements anticancéreux : la cryopréservation des gamètes et du tissu gonadique.

Manuscrit reç : juillet 2004 ; accepté septembre 2004.

\section{ABSTRACT}

Regulatory and organizational aspects of cryopreservation. The jurist's point of view

\section{Anna GRABINSKI}

At the present time, legal texts in application of bioethics laws only briefly mention cryopreservation. The conditions of cryopreservation differ according to the type of tissue stored: while cryopreservation of oocytes and ovarian tissue corresponds to the field of biomedical research, semen cryopreservation corresponds to medically assisted procreation.
Cryopreservation activity is more clearly defined in the draft revision of the bioethics law.

Concerning the cryoconservation of ovarian tissue and oocytes, these difficulties result in particular in this activity's mixed nature. It is located indeed, halfway between research and care. These two spheres of medical activity are subjected to their own distinct and exclusive, and their application is conditioned by the qualification of the implemented act.

However, this qualification is dubious here, because of, in particular, the impossibility of determining which acts of sampling, cryoconservation and use might be concerned with a research protocol.

Concerning the cryoconservation of sperm, the texts, first of all, seem to assimilate the activity of cryoconservation within an activity of assisted medical procreation.

However, such assimilation would be equivalent to the impossibility of its implementation, because of the difficulty of respecting all of the legal conditions of assisted medical procreation. However, another more favourable interpretation of the provisions seems to be possible.

Taking into consideration these uncertainties, contradictions and difficulties, the legislator intervened and devoted, by the widening of the indications of assisted medical procreation, the activity of cryoconservation in the project of revision of the laws of bioethics. He however did not solve all the difficulties.

These cryoconserved elements can be restored only to the depositor. Indeed, the texts make obstacle to a delivery for a third party of the cryoconserved sperm, whatever the moment.

They can also be used. This use can consist of assisted medical procreation, which is the first finality of cryoconservation - but it could only be implemented in respect of the whole of the legal conditions.

It seems that the cryoconserved elements could also be used within the framework of research, whatever its nature (biomedical or not) and the moment of its implementation (while the person is alive or after his death).

Its implementation should however be subjected to prior agreement and expressed while the person, whose elements were preserved, is alive. Such a use is expressly made possible in the project of revision of the laws of bioethics.

Following a partial use or a lack of such a use, the destruction of the cryoconserved elements can be considered, as well as the continuation of the cryoconservation.

However, these hypotheses raise difficulties that have not yet been resolved in the draft revision of the bioethics law.

Key words : sperm straw, oocytes, ovarian tissue, biomedical research, assisted medical procreation, restitution, destruction, continuation of the conservation 


\section{NOTES}

1 La loi relative à la bioéthique a été définitivement adoptée. II s'agit de la loi $n^{\circ} 2004-800$ du 6 août 2004, publiée au JO n'182 du 7 août 2004, p. 14040 et s.

2 Point 2.6 de l'arrêté du 12 janvier 1999: "Ce chapitre concerne essentiellement l'autoconservation de sperme. En effet, la congélation d'ovocytes et les prélèvements de fragments d'ovaire en vue de conservation pour une éventuelle AMP ultérieure restent du domaine de la recherche et doivent donc entrer dans le cadre d'un protocole de recherche et faire l'objet d'une demande à un CCPPRB. "

3 Art. L. 2141-11 du projet : « En vue de la réalisation ultérieure d'une assistance médicale à la procréation, toute personne peut bénéficier du recueil et de la conservation de ses gamètes ou de tissu germinal, avec son consentement et, le cas échéant, celui de l'un des titulaires de l'autorité parentale, ou du tuteur lorsque l'intéressé mineur ou majeur fait l'objet d'une mesure de tutelle, lorsqu'une prise en charge médicale est susceptible d'altérer sa fertilité, ou lorsque sa fertilité risque d'être prématurément altérée. "

4 On peut dès lors s'interroger sur la compatibilité de cet article avec l'article L.1110-5 du CSP, issu de la loi du 4 mars 2002, selon lequel " toute personne a le droit, compte tenu de son état de santé et de l'urgence des interventions que celui-ci requiert, de recevoir les soins les plus appropriés et de bénéficier des thérapeutiques dont l'efficacité est reconnue et qui garantissent la meilleure sécurité sanitaire au regard des connaissances médicales avérées. Les actes de prévention, d'investigation ou de soins ne doivent pas, en l'état des connaissances médicales, lui faire courir des risques disproportionnés par rapport au bénéfice escompté". Quel pourrait être, dans cette hypothèse, le résultat de la balance risques-bénéfices?

5 Article L.2141-2 CSP :

«L'assistance médicale à la procréation est destinée à répondre à la demande parentale d'un couple.

Elle a pour objet de remédier à l'infertilité dont le caractère pathologique a été médicalement diagnostiqué. Elle peut aussi avoir pour objet d'éviter la transmission à l'enfant d'une maladie d'une particulière gravité.

L'homme et la femme formant le couple doivent être vivants, en âge de procréer, mariés ou en mesure d'apporter la preuve d'une vie commune d'au moins deux ans et consentants préalablement au transfert des embryons ou à l'insémination. "

6 N'apparaît pas souligné dans le texte (ndla)

7 Article L.1211-2 du projet : " L'utilisation d'éléments et de produits du corps humain à une fin médicale ou scientifique autre que celle pour laquelle ils ont été prélevés ou collectés est possible, sauf opposition exprimée par la personne sur laquelle a été opéré ce prélè- vement ou cette collecte, dûment informée au préalable de cette autre fin. Lorsque cette personne est un mineur ou un majeur sous tutelle, l'opposition est exercée par les titulaires de l'autorité parentale ou le tuteur. II peut être dérogé à l'obligation d'information lorsque celle-ci se heurte à l'impossibilité de retrouver la personne concernée, ou lorsqu'un des comités consultatifs de protection des personnes mentionnés à l'article L. 1123-1, consulté par le responsable de la recherche, n'estime pas cette information nécessaire. Toutefois, ces dérogations ne sont pas admises lorsque les éléments initialement prélevés consistent en des tissus ou cellules germinaux. Dans ce dernier cas, toute utilisation pour une fin autre que celle du prélèvement initial est interdite en cas de décès de l'intéressé. "

8 La nouvelle fin scientifique ne consistera pas en une recherche biomédicale. En effet, dans la rédaction actuelle de la loi Huriet, seuls entrent dans son champ d'application les actes qui, d'une part, sont susceptibles de porter directement atteinte à l'intégrité physique de la personne et, d'autre part, qui peuvent avoir une répercussion sur la santé de la personne qui s'y prête. De ce fait, les recherches effectuées sur des échantillons déjà prélevés ne sauront être considérés comme des recherches biomédicales.

Elles ne seront pas non plus considérées comme une recherche biomédicale une fois adoptées les dispositions modifiées de la loi Huriet (Projet de loi relatif à la santé publique, adopté en première lecture par le Sénat Nationale le 19 janvier 2004). Ces dispositions prévoient expressément l'exclusion des études interventionnelles du champ d'application de la loi Huriet - en effet, en vertu de l'article L.1121-1 alinéa 2 modifié, " les dispositions du présent titre ne s'appliquent pas aux recherches dans lesquelles tous les actes sont pratiqués et les produits utilisés de manière habituelle et lorsque aucune procédure supplémentaire ou inhabituelle de diagnostic ou de surveillance n'est appliquée (...)" ". 